\title{
ARTICLE
}

\section{A preliminary study on streaming effect through the gap of blanket modules for a K-DEMO fusion reactor}

\author{
Song Hyun Kimª , Ji Sung Park ${ }^{\mathrm{a}}$, Chang-ho Shin ${ }^{\mathrm{a}}$, Young Seok Lee ${ }^{\mathrm{b}}$ and Jong Kyung Kim ${ }^{\mathrm{a}}$ \\ ${ }^{a}$ Department of Nuclear Engineering, Hanyang University, 222 Wangsimni-ro, Seongdong-gu, Seoul 133-791, Korea; \\ ${ }^{b}$ National Fusion Research Institute (NFRI), Gwahak-ro, Yuseong-gu, Daejeon 305-806, Korea
}

\begin{abstract}
It is known that the blanket module in the fusion reactor is designed for slowing and absorbing neutrons, breeding tritium, and limiting radiation damage to other structures. These blankets are installed remotely at individual positions. In this process, gaps between the blanket modules can occur and the streaming effect through gaps has an effect on the performance of the fusion reactor. The streaming effect affects two aspects in the neutron transport problem: (1) activation of the fusion reactor structures and (2) radiation shielding. It is noted that the shape of the gap is locally different due to the specific shape of the fusion reactor. Also, the mechanical difference of the blanket modules changes the gap size. In this study, the streaming effect caused by different shape and size of the gap are evaluated for a K-DEMO fusion reactor. The blanket modules of the fusion reactor, which are the blanket, vacuum vessel, and water channel, were simply simulated. In each module, the neutron streaming effects were assessed by changing the gap size and shapes. The MCNP5 code was used for the evaluations with ENDF/B-VI cross-section library and SAB2002 thermal cross section library. It was noticed that the neutron flux was significantly changed due to the streaming effect. This study can give guidelines for the simplification of blanket modeling in radiation transport problems.
\end{abstract}

Keywords: K-DEMO fusion reactor; streaming effect; gap of blanket; Monte Carlo; neutron flux distribution; radiation shielding

\section{Introduction}

K-DEMO fusion reactor is under conceptual design in Korea for testing of the commercial level fusion reactor [1]. The blanket modules, which are directly contacted with the reactor core, are designed for moderating and absorbing neutrons, breeding tritium, and limiting radiation damage to other structures. Each blanket module is remotely installed at an individual position. In this process, the gaps between the blanket modules occur and the streaming [2, 3] through the gap affects the performance of the fusion reactor. The fusion reactor is a large facility; hence, the neutron flux evaluation at the local region is difficult with Monte Carlo method. To solve the problem, there is a future plan to use a simplified model of the fusion reactor without the gap modeling. However, the streaming effect cannot be considered in the simplified model. In this study, the streaming effects are evaluated for three aspects to offer the information of the streaming effect in using the simplified fusion reactor model: (1) change of the overall gap sizes, (2) change of the partial gap sizes, and (3) the gap shape change.

*Corresponding author. Email: jkkim1@hanyang.ac.kr

\section{Methodology}

\subsection{Overview of blanket module and shielding}

Two kinds of the blanket modules, which are called inboard-side blanket (IB) and outboard-side blanket (OB), are installed in the K-DEMO fusion reactor. The IB and OB are located at Region A and Region B as shown in Figure 1, respectively. The IB has $100 \mathrm{~cm} \mathrm{x}$ $100 \mathrm{~cm}$ x $55.2 \mathrm{~cm}$ cuboid structure and 40 layers while OB has $100 \mathrm{~cm} \times 100 \mathrm{~cm} \times 67.2 \mathrm{~cm}$ cuboid structure and 52 layers. The design details of the IB and OB are given in Table 1 and 2.

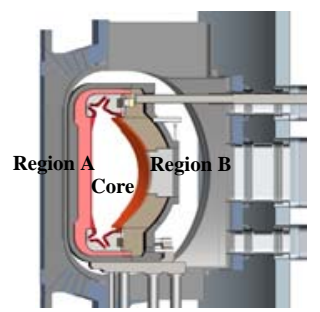

Figure 1. Vertical view of K-DEMO fusion reactor [1].

The core is surrounded by the shielding which is located outside of the blanket modules. The thicknesses of the inboard-side shielding and outboard-side shielding are $10 \mathrm{~cm}$ and $20 \mathrm{~cm}$, respectively. Both shields are 
composed of $\mathrm{B}_{4} \mathrm{C}$ material.

\subsection{Evaluation method for streaming effect}

The neutron streaming effect through the blanket gaps leads to the increase of the local flux as well as the average flux. There are two major aspects to evaluate the streaming effect with Monte Carlo method. Firstly, Monte Carlo method uses the stochastic method with random sampling of the each particle; therefore, it is difficult to directly evaluate the local flux for the large size facility such as the fusion reactor. Secondly, the gaps can have various shapes and sizes in the blanket locations due to the specific shape of reactor and mechanical difference. In this study, a hexahedron model as shown in Figure 2 is proposed to evaluate the relative flux variations caused by the gap streaming. The reflective boundaries were used as shown in Figure 3. It is assumed that the gaps are located at the bottom and the left side of the blanket module. Also, it is assumed that the neutron source, which has Gaussian fusion energy spectrum and isotropic angular source, is uniformly located at the front side of the hexahedron model. The streaming effects for both blanket types were evaluated with changes of the gap sizes, the partial gap sizes and the gap shapes. The MCNP5 code [4] was used for the calculations with ENDF/B-VI cross-section library and SAB2002 thermal cross section library.

MCNP modeling for both types of the blanket modules were performed as shown in Figure 4. The tallies for the flux evaluations were located at the front and backward surfaces of the shielding (S-I and S-II) and the cell of the shield (C-I) as shown in the Figure 4.

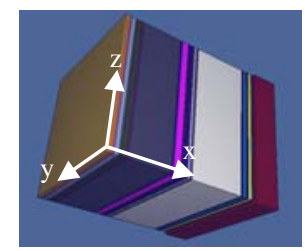

Figure 2. Hexahedron model for the streaming effect analysis.

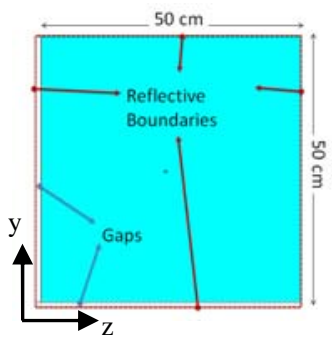

Figure 3. Radial view of hexahedron model.

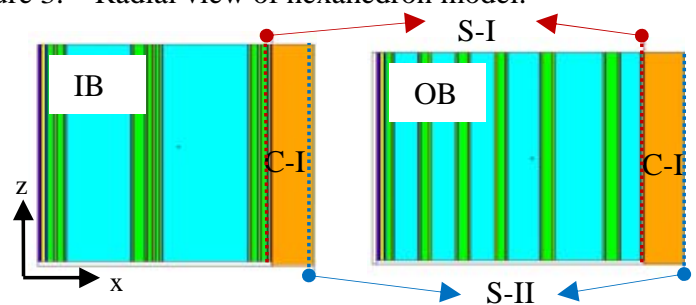

Figure 4. MCNP modeling of IB and OB.
Table 1. Design details of IB [1].

\begin{tabular}{lcc}
\hline \multicolumn{1}{c}{ Layer } & Thickness $(\mathrm{cm})$ & Material \\
\hline FW & 0.5 & Tungsten \\
Cooling Channel & $0.5 / 0.8 / 0.5$ & SUS316/Water/ SUS316 \\
1st Breeder & 1.5 & Li4SiO4 (60\% enriched Li-6) \\
Cooling Channel & $0.2 / 0.4 / 0.2$ & SUS316/Water/ SUS316 \\
2nd Breeder & 1.5 & Li4SiO4 (60\% enriched Li-6) \\
Cooling Channel & $0.2 / 0.4 / 0.2$ & SUS316/Water/ SUS316 \\
1st Be & 15 & Be \\
Cooling Channel & $0.2 / 0.4 / 0.2$ & SUS316/Water/ SUS316 \\
3rd Breeder & 2.5 & Li4SiO4 (60\% enriched Li-6) \\
Cooling Channel & $0.2 / 0.4 / 0.2$ & SUS316/Water/ SUS316 \\
4th Breeder & 1 & Li4SiO4 (60\% enriched Li-6) \\
Cooling Channel & $0.2 / 0.4 / 0.2$ & SUS316/Water/ SUS316 \\
5th Breeder & 1 & Li4SiO4 (60\% enriched Li-6) \\
Cooling Channel & $0.2 / 0.4 / 0.2$ & SUS316/Water/ SUS316 \\
2nd Be & 20 & Be \\
Cooling Channel & $0.2 / 0.4 / 0.2$ & SUS316/Water/ SUS316 \\
6th Breeder & 1.5 & Li4SiO4 (60\% enriched Li-6) \\
Cooling Channel & $0.2 / 0.4 / 0.2$ & SUS316/Water/ SUS316 \\
7th Breeder & 1.5 & Li4SiO4 (60\% enriched Li-6) \\
Cooling Channel & $0.3 / 0.4 / 0.3$ & SUS316/Water/ SUS316 \\
\hline
\end{tabular}

Table 2. Design details of the OB [1].

\begin{tabular}{lcc}
\hline \multicolumn{1}{c}{ Layer } & Thickness $(\mathrm{cm})$ & Material \\
\hline FW & 0.5 & Tungsten \\
Cooling Channel & $0.6 / 0.8 / 0.4$ & SUS316/Water/ SUS316 \\
1st Breeder & 1.5 & Li4SiO4 (40\% enriched Li-6) \\
Cooling Channel & $0.15 / 0.4 / 0.15$ & SUS316/Water/ SUS316 \\
1st Be & 6 & Be \\
Cooling Channel & $0.15 / 0.4 / 0.15$ & SUS316/Water/ SUS316 \\
2nd Breeder & 2 & Li4SiO4 (40\% enriched Li-6) \\
Cooling Channel & $0.15 / 0.4 / 0.15$ & SUS316/Water/ SUS316 \\
2nd Be & 6 & Be \\
Cooling Channel & $0.15 / 0.4 / 0.15$ & SUS316/Water/ SUS316 \\
3rd Breeder & 2.5 & Li4SiO4 (40\% enriched Li-6) \\
Cooling Channel & $0.15 / 0.4 / 0.15$ & SUS316/Water/ SUS316 \\
3rd Be & 6 & Be \\
Cooling Channel & $0.15 / 0.4 / 0.15$ & SUS316/Water/ SUS316 \\
4th Breeder & 2 & Li4SiO4 (40\% enriched Li-6) \\
Cooling Channel & $0.15 / 0.4 / 0.15$ & SUS316/Water/ SUS316 \\
4th Be & 8 & Be \\
Cooling Channel & $0.15 / 0.4 / 0.15$ & SUS316/Water/ SUS316 \\
5th Breeder & 2.5 & Li4SiO4 (40\% enriched Li-6) \\
Cooling Channel & $0.15 / 0.4 / 0.15$ & SUS316/Water/ SUS316 \\
5th Be & 12 & Be \\
Cooling Channel & $0.15 / 0.4 / 0.15$ & SUS316/Water/ SUS316 \\
6th Breeder & 3 & Li4SiO4 (40\% enriched Li-6) \\
Cooling Channel & $0.15 / 0.4 / 0.15$ & SUS316/Water/ SUS316 \\
6th Be & 5 & Be \\
Cooling Channel & $0.3 / 0.4 / 0.3$ & SUS316/Water/ SUS316 \\
\hline
\end{tabular}

\section{Result and discussion}

\subsection{Streaming effect with various gap sizes}

The evaluation of the neutron flux was pursued with the changes of the gap sizes from $1.0 \mathrm{~cm}$ to $3.0 \mathrm{~cm}$ by increasing $0.1 \mathrm{~cm}$ with considering the gap size of ITER fusion reactor [2]. To estimate how the streaming affects the average flux, the relative differences compared with the results of a case, which has no gaps between the blankets (0 gap), were calculated on the surfaces S-I and S-II, respectively. The results of the relative differences are given in Table 3 . The maximum relative difference was $276.28 \%$ at S-II of OB with $3 \mathrm{~cm}$ gaps. To estimate the variation of the local flux distribution caused by the streaming effect, C-I cells were divided into the $10 \times 10$ equal sub-regions. The calculations of the local fluxes were performed with the $2 \mathrm{~cm}$ gaps. The results of the flux distributions are given in Figure 5. The peak fluxes at the C-I cells of the IB and OB were $229.50 \%$ and $284.28 \%$ higher than the average fluxes, respectively. The relative differences of the neutron energy distributions at S-I surfaces were estimated for study of how the streaming effect can affect the activation analysis. The results are given as shown in Figure 6. 
Table 3. Relative differences of average fluxes on surfaces S-I and S-II compared with '0 Gap' case.

\begin{tabular}{|c|c|c|c|c|}
\hline \multirow{2}{*}{ Gap Size } & \multicolumn{2}{|c|}{ IB } & \multicolumn{2}{c|}{ OB } \\
\cline { 2 - 5 } & S-I & S-II & S-I & S-II \\
\hline $1.0 \mathrm{~cm}$ & $15.53 \%$ & $20.61 \%$ & $25.34 \%$ & $40.09 \%$ \\
\hline $1.1 \mathrm{~cm}$ & $17.73 \%$ & $23.69 \%$ & $28.94 \%$ & $46.19 \%$ \\
\hline $1.2 \mathrm{~cm}$ & $19.97 \%$ & $26.83 \%$ & $33.03 \%$ & $53.95 \%$ \\
\hline $1.3 \mathrm{~cm}$ & $22.29 \%$ & $30.11 \%$ & $37.12 \%$ & $61.04 \%$ \\
\hline $1.4 \mathrm{~cm}$ & $24.72 \%$ & $33.78 \%$ & $41.74 \%$ & $69.02 \%$ \\
\hline $1.5 \mathrm{~cm}$ & $27.34 \%$ & $37.43 \%$ & $45.79 \%$ & $78.23 \%$ \\
\hline $1.6 \mathrm{~cm}$ & $29.91 \%$ & $41.32 \%$ & $50.80 \%$ & $86.27 \%$ \\
\hline $1.7 \mathrm{~cm}$ & $32.59 \%$ & $45.61 \%$ & $55.81 \%$ & $98.56 \%$ \\
\hline $1.8 \mathrm{~cm}$ & $35.38 \%$ & $49.83 \%$ & $61.36 \%$ & $107.92 \%$ \\
\hline $1.9 \mathrm{~cm}$ & $38.19 \%$ & $54.38 \%$ & $67.34 \%$ & $119.85 \%$ \\
\hline $2.0 \mathrm{~cm}$ & $40.94 \%$ & $58.47 \%$ & $73.29 \%$ & $133.14 \%$ \\
\hline $2.1 \mathrm{~cm}$ & $43.83 \%$ & $63.45 \%$ & $78.81 \%$ & $146.07 \%$ \\
\hline $2.2 \mathrm{~cm}$ & $46.79 \%$ & $68.05 \%$ & $85.15 \%$ & $156.86 \%$ \\
\hline $2.3 \mathrm{~cm}$ & $50.08 \%$ & $72.82 \%$ & $91.63 \%$ & $171.29 \%$ \\
\hline $2.4 \mathrm{~cm}$ & $53.04 \%$ & $77.98 \%$ & $97.77 \%$ & $185.10 \%$ \\
\hline $2.5 \mathrm{~cm}$ & $56.38 \%$ & $83.12 \%$ & $104.54 \%$ & $197.54 \%$ \\
\hline $2.6 \mathrm{~cm}$ & $59.76 \%$ & $88.64 \%$ & $111.60 \%$ & $213.49 \%$ \\
\hline $2.7 \mathrm{~cm}$ & $63.04 \%$ & $94.06 \%$ & $118.39 \%$ & $228.20 \%$ \\
\hline $2.8 \mathrm{~cm}$ & $66.64 \%$ & $99.89 \%$ & $126.15 \%$ & $245.10 \%$ \\
\hline $2.9 \mathrm{~cm}$ & $70.11 \%$ & $105.16 \%$ & $133.57 \%$ & $258.99 \%$ \\
\hline $3.0 \mathrm{~cm}$ & $73.61 \%$ & $111.46 \%$ & $141.16 \%$ & $276.28 \%$ \\
\hline
\end{tabular}

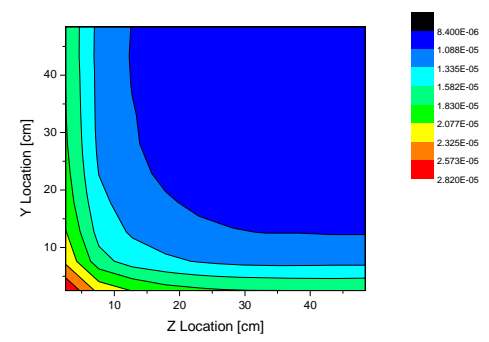

(a) Local flux distribution in C-I of IB

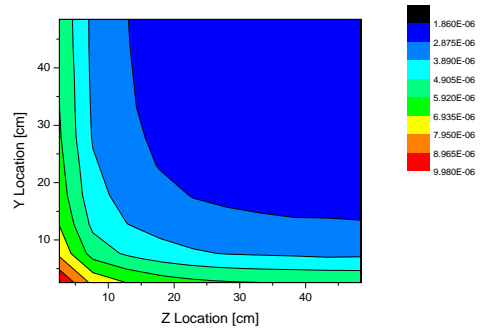

(b) Local flux distribution in C-I of OB

Figure 5. Local flux distributions with $2 \mathrm{~cm}$ gap at C-I cell.

The maximum relative differences of IB and OB cases were $765.75 \%$ and $3429.65 \%$ at the highest energy level, respectively. It is understood that the increases of the high energy neutrons were caused by the direct leakages of the source neutrons through the gaps.

\subsection{Streaming effect with partial gap differences}

The streaming effect with the gap changes, which is caused by the mechanical difference of the blanket modules, was evaluated with a model as shown in Figure 7. It is assumed that the designed gap size is 2 $\mathrm{cm}$. Also, it is assumed that one of the gap sizes is changeable due to the mechanical difference. The maximum range of the gap difference is assumed to be $20 \%( \pm 4 \mathrm{~mm})$ which is a conservative limit of the mechanical difference. The relative differences compared with the $2 \mathrm{~cm}$ gap size are shown in Figure 8.

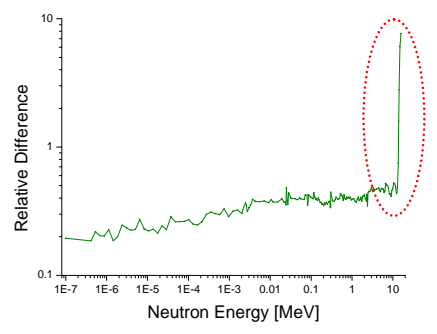

(a) Neutron energy distribution at S-I of IB

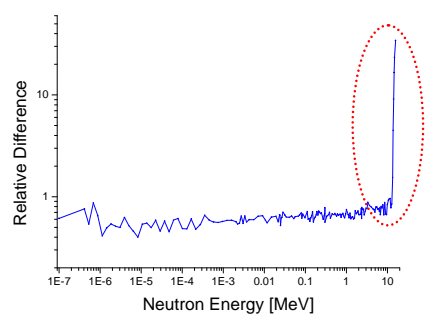

(b) Neutron energy distribution at S-I of OB

Figure 6. Relative difference of neutron energy distributions with $2 \mathrm{~cm}$ gaps at S-I surfaces of IB and OB.

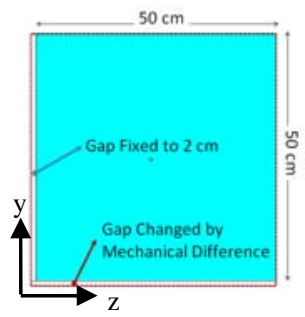

Figure 7. Description of MCNP modeling for the streaming effect analysis of the gap differences.

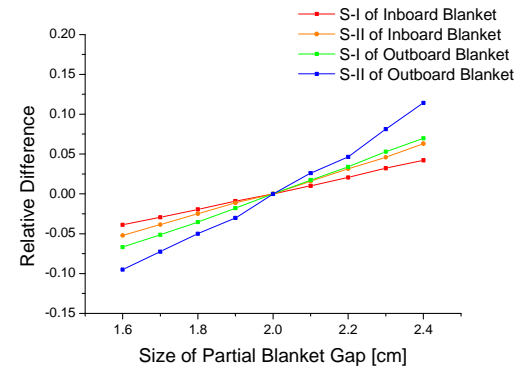

Figure 8. Relative differences of neutron fluxes by changing the partial gap size.

The maximum relative differences of the neutron fluxes in the IB and OB were $6.30 \%$ and $11.42 \%$ compared with $2 \mathrm{~cm}$ gaps at S-II surfaces, respectively. To analyze the local flux variations caused by mechanical difference, the local flux were evaluated by dividing C-I cells into the $10 \times 10$ sub-regions. Also, it is assumed that the gap size is $2 \mathrm{~cm}$, and one of the gap sizes is increased to $2.2 \mathrm{~cm}$ as a result of the mechanical difference. Figure 9 shows the results of the neutron flux distributions. The results show that the local pick fluxes at the C-I cells of the IB and OB were increased to $5.55 \%$ and $8.38 \%$ compare with Figure 6 results, respectively. 


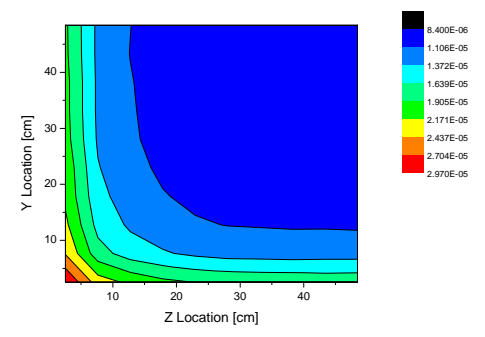

(a) Local flux distribution in C-I of IB

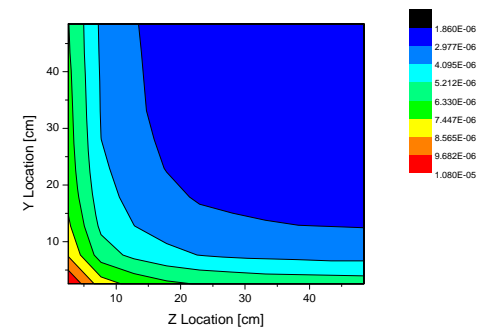

(b) Local flux distribution in C-I of OB

Figure 9. Neutron flux distributions with partial gap increase.

\subsection{Streaming with gap shapes}

Due to the fusion reactor design, the gap shapes are different. In this study, a simple model for the streaming effect analyses with various gap shapes is proposed as shown in Figure 10. The gradients of bottom planes are assumed to be $-5^{\circ},-1^{\circ}, 1^{\circ}$ and $5^{\circ}$ gradients based on $\mathrm{x}-\mathrm{y}$ plane. The average neutron fluxes with the model are given as shown in Figure 11. The maximum fluxes for the IB and OB were appeared at $-5^{\circ}$ cases with $160.51 \%$ and $243.47 \%$ increases than that of the $0^{\circ}$ case.

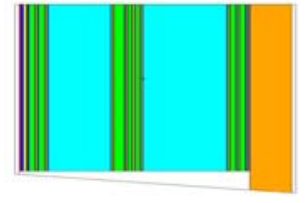

(a) $-5^{\circ}$ gradient case of IB

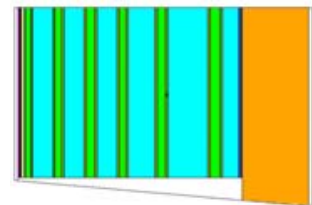

(c) $-5^{\circ}$ gradient case of $\mathrm{OB}$

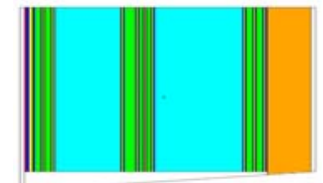

(b) $5^{\circ}$ gradient case of IB

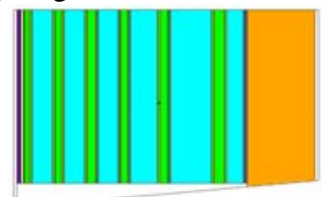

(d) $5^{\circ}$ gradient case of $\mathrm{OB}$
Figure 10. MCNP modeling for the streaming effect analysis with the gap shape change.

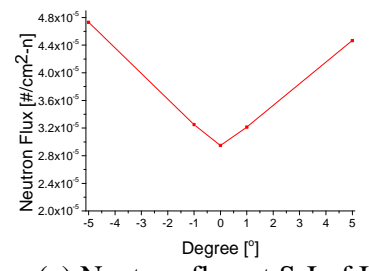

(a) Neutron flux at S-I of IB

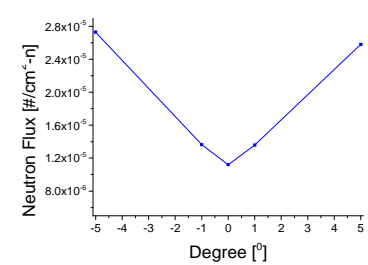

(b) Neutron flux at S-I of OB
Figure 11. Results of average fluxes with changes of the gap angles.

\section{Conclusion}

In this study, the streaming effect through the gaps between blanket modules was evaluated. The streaming effect was evaluated with three aspects which are the size of the gap, the local gap size change caused by mechanical difference, and specific shapes of the gaps. Analysis shows that the streaming effect significantly affects not only the average fluxes, but also the local flux distribution. Also, these results show that the streaming effect must be properly considered for the neutron transport calculations. It is expected that the analysis of the streaming effect can offer reference data for the activation and shielding evaluations in the fusion reactor. In addition, this study can give a guideline for the simplification of the blanket modeling in radiation transport problems.

\section{Future work}

In this study, the relative effect of the gap streaming was analyzed. After the finish of the K-DEMO reactor design, following evaluations will be performed.

1) Full and Detail Reactor Modeling of the K-DEMO

2) Data Construction of the Local Flux Variations with a Single Blanket Model including the Gap

3) Evaluation of the Average Flux by using the Full Reactor Model

4) Correction of the Average Flux by using the Data from Step 2

\section{Acknowledgements}

This work was supported in part by the Ministry of Education, Science and Technology of Korea through the project for the radiation effect on fusion reactor coordinated by NFRI (2012-00000000-680) and Innovative Technology Center for Radiation Safety (iTRS) at Hanyang University.

\section{References}

[1] Advanced Project Division, K-DEMO Study Plan, National Fusion Research Institute (NFRI), May 8th (2012).

[2] S. Sato, H. Takatsu, H. Iida, S. Mori and R. Santoro, streaming analysis for radiation through ITER mid-plane port, Fusion Energy and Design 42 (1998), pp. 213-219.

[3] S. Sato, H. Iida, R. Plenteda and R. T. Santoro, Monte Carlo analysis of helium production in the ITER shield blanket module, Fusion Energy and Design 46 (1999), pp. 1-9.

[4] X-5 Monte Carlo Team, MCNP-A General Monte Carlo N-Particle Transport Code, Version 5, Volume II: User's Guide, LA-CP-03-0245, Los Alamos National Laboratory, (2003). 